\section{TESES DE DOUTORADO EM GESTÃO DO ESPORTE NO BRASIL: UMA REVISÃO INTEGRATIVA METODOLÓGICA}

\author{
DOCTORAL THESES IN SPORT MANAGEMENT IN BRAZIL: AN \\ INTEGRATIVE METHODOLOGICAL REVIEW C?
}

TESIS DOCTORALES EN GESTIÓN DEPORTIVA EN BRASIL: UNA

REVISIÓN INTEGRADORA METODOLÓGICA Cें

doi' https://doi.org/10.22456/1982-8918.103915

(D) Ivan Furegato Moraes* <ifuregato@gmail.com>

Cacilda Mendes dos Santos Amaral** <cacilda.amaral@uemg.br>

Flávia da Cunha Bastos*<flaviacb@usp.br>

\author{
*Universidade de São Paulo. São Paulo, SP, Brasil. \\ **Universidade do Estado de Minas Gerais. Divinópolis, MG, Brasil.
}

\begin{abstract}
Resumo: O presente estudo teve como objetivo analisar temáticas e métodos de pesquisa na produção de teses de doutorado em Gestão do Esporte em programas de pós-graduação de IES brasileiras. A pesquisa, de caráter exploratório, descritivo e abordagem mista, foi realizada através de revisão integrativa do tipo metodológica, considerando-se teses defendidas até 2019. Foram identificadas 94 teses, a partir de 1997, com distribuição geográfica desigual no país, majoritariamente produzidas em universidades públicas, em programas de Educação Física e Administração. As teses apresentam diversidade de temáticas, com predomínio de políticas públicas e lacunas quanto a outros temas de pesquisa. Revelaram-se aspectos críticos quanto à estrutura metodológica, com falta de informações quanto à abordagem e aos métodos de pesquisa e de análise. Conclui-se que o panorama da produção acadêmica sobre a área revela defasagens coerentes com as do desenvolvimento científico da área no país.
\end{abstract}

Palavras chave: Gestão. Esportes. Teses Eletrônicas. Indicadores de Produção Científica.
Recebido em: 04 jun. 2020 Aprovado em: 08 dez. 2020 Publicado em: 18 fev. 2021

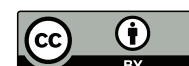

Este é um artigo publicado sob a licença Creative Commons Atribuição 4.0 Internacional (CC BY 4.0). elSSN: $1982-8918$ 


\section{INTRODUÇÃO}

As teorias que norteiam a atuação de gestores de organizações esportivas estão, na maioria das vezes, pautadas na aplicação de conceitos da Administração (CHELLADURAI, 2013; PARKHOUSE, 2005; SLACK, 2014). A definição de Administração do Esporte, ou Gestão do Esporte, é trabalhar ou coordenar pessoas e recursos de forma que atinjam objetivos da organização esportiva de forma eficaz (FRANCALACCI, 2011; ROCHA; BASTOS, 2011). Segundo Francalacci (2011), o termo Administração Esportiva é a denominação histórica da área no Brasil, já o termo Gestão do Esporte (GE) tem sido apontado como o mais apropriado atualmente no país (ABRAGESP, 2009; FRANCALACCI, 2011; ROCHA; BASTOS, 2011).

Tanto a Ciência do Esporte como a Administração têm considerado a GE como área de conhecimento nova, disciplina híbrida, multidisciplinar, sendo desenvolvida majoritariamente em escolas de negócios ou em escolas de Educação Física e Esporte (CHALIP, 2006). Este campo de estudo tem buscado a legitimidade a partir da relação com outras disciplinas de referência, que fornecem conhecimentos fundamentais para que a GE progrida (SHILBURY, 2011), como Administração, Marketing, Economia, Psicologia, Lazer, Turismo e a própria Educação Física (CIOMAGA, 2013; SLACK, 2014).

Quanto ao desenvolvimento da área, para a sua solidificação como um campo legítimo e com credibilidade, são necessários profissionais preparados, organizações dedicadas ao avanço da área, corpo de conhecimento e literatura própria em relação à teoria e à prática e o desenvolvimento de pesquisas com rigor científico (CHALIP, 2006; CHELLADURAI, 2013; PITTS, 2001). Essa visão, apresentada no início do século XXI, ainda permanece válida, sobretudo em regiões e países nos quais a ciência da GE está em níveis iniciais de produção científica.

O ensino e a pesquisa em GE têm sido desenvolvidos de forma diferenciada em diversas partes do mundo. Estados Unidos e Europa possuem, há décadas, programas acadêmicos, da graduação ao doutorado; promovem, através de associações nacionais e continentais, congressos e outros eventos científicos, e mantêm periódicos científicos com níveis de excelência (PIRES; SARMENTO, 2001; PITTS; DANYLCHUCK; QUARTERMAN, 2014; SAJJADI et al., 2013; SCELLES, 2020).

Com o crescimento daárea, o foco se voltou para o rigor e a qualidade acadêmica dos programas, fazendo-se necessário o credenciamento e a prestação de contas sobre a educação e o ensino em GE (YIAMOUYIANNIS et al., 2013). Na América do Norte há um conselho na North American Society for Sport Management (NASSM) responsável pela excelência no ensino da GE nas Instituições de Ensino Superior (IES), do bacharelado à pós-graduação, que coordena uma comissão especializada, a Commission on Sport Management Accreditation (COSMA) (COSMA, 2016).

O processo de reconhecimento e credenciamento dos cursos em GE é rigoroso, abrangente, revisado por pares e projetado para melhorar a qualidade da educação em instituições legítimas, com intuito de manter a qualidade do ensino e impedir a proliferação das chamadas "fábricas de diplomas", ou seja, instituições de ensino pouco comprometidas com a formação dos profissionais e que ofereceriam 
uma formação mais rápida, barata e de má qualidade (YIAMOUYIANNIS et al., 2013). A última revisão dos componentes, publicada em 2016, elenca cinco áreas de formação mínimas principais: Fundamentos do Esporte: Históricos, Sociológicos e Psicológicos; Fundamentos, Funções e Ambiente da Gestão do Esporte; Experiências Integrativas; e Plano de Carreira.

A formação em GE na América do Norte reverbera na produção acadêmica. Em revisão de literatura sobre o Ensino Superior em GE, Miragaia e Soares (2017) apontam que entre 1979 e 2014 a produção de maior impacto na área de ensino da GE provém de autores filiados a instituições dos Estados Unidos (MIRAGAIA; SOARES, 2017). Entretanto, segundo Dowling (2018), ainda existe um número limitado de oportunidades para aqueles interessados em buscar um doutorado ou pós-doutorado na área, afetando de forma significativa a sua consolidação em termos de produção acadêmica.

Estudos que analisaram pesquisas publicadas nos principais periódicos da área apontam para o desenvolvimento mais proeminente de pesquisas oriundas de doutorados nos Estados Unidos, Europa e Austrália. Há uma tendência de maior número de pesquisas sobre as temáticas Marketing Esportivo, Economia do esporte, aspectos gerenciais e organizacionais do esporte, responsabilidade social e sobre gestão de eventos esportivos (CIOMAGA, 2013; LARA, 2014; PITTS; DANYLCHUCK; QUARTERMAN, 2014; PITTS; PEDERSEN, 2005; SHILBURY, 2011). Os autores também apontam áreas pouco exploradas, como Ética, Direito, Finanças, Comunicação e experiências de campo, demonstrando que a GE ainda se mantém como um campo emergente em termos de estabelecimento de uma base de conhecimento internamente consistente (DOWLING, 2018).

Recentemente, Scelles (2020) analisou a produção sobre GE em língua inglesa, identificando temáticas em diferentes periódicos a partir de 2014. O autor aponta sete categorias que englobam 21 temáticas recorrentes, base para propor uma Agenda 21 para a área, agrupados em sete categorias (três por categoria): Realidade do esporte no século XXI; Políticas públicas e política esportiva; Necessidade de mudanças; Adaptação, Criação e Otimização; Público Negligenciado e/ou Específico; Esportes, estados e eventos negligenciados; Reconhecimento da área (SCELLES, 2020).

Entendendo que a consolidação das pesquisas e a produção de conhecimento de alto impacto estão relacionados à robustez e à confiabilidade do desenho metodológico, Pitts e Pedersen (2005) analisaram a metodologia empregada nos estudos publicados no Journal of Sport Management, periódico tradicional sobre GE. Foi verificada a predominância de pesquisas com abordagem quantitativa (68\%), com 18\% utilizando Estatística Descritiva e as demais, Estatística Inferencial, especialmente a Análise Multivariada de Dados. Sobre os de abordagem qualitativa, $50 \%$ eram de caráter descritivo (PITTS; PEDERSEN, 2005).

Para Edwards e Skinner (2009), como a GE ainda é uma área em ascensão, seria desejado um maior número de pesquisas de abordagem qualitativa, haja vista o desconhecimento que existe sobre este campo de estudo. Além disso, outras análises têm recomendado a aplicação de metodologias de pesquisa inovadoras, relevantes e flexíveis, essenciais para o desenvolvimento científico da área (AMIS; SILK, 2005; SKINNER; ENGELBERG, 2018; SMITH; STEWART, 2010). 
No Brasil, a GE tem seu desenvolvimento acentuado em termos acadêmicos e científicos a partir dos anos 2000 (BASTOS, 2019; BASTOS; CASTRO; AMARAL, 2015; FRANCALACCI, 2011; ROCHA; BASTOS, 2011). Apesar de haver cursos de formação em nível de graduação (tecnólogo) no Brasil, não há uma diretriz única para formação dos profissionais, ou seja, os currículos e cursos oferecidos possuem diferentes formatos e objetivos (MAZZEI; AMAYA; BASTOS, 2013).

No que diz respeito à pós-graduação, não há cursos específicos no Brasil, seja em nível de mestrado (acadêmico ou profissional) ou de doutorado. Entretanto, diferentes IES tem acolhido pesquisadores de temáticas ligadas à GE em programas de diferentes áreas do conhecimento (BASTOS, 2016; GAYA et al., 2018).

O desenvolvimento de estudos mais robustos e com rigor científico muitas vezes está relacionado à produção dos programas de pós-graduação das IES, principalmente por meio da produção de dissertações e teses (ALMEIDA; GUIMARÃES, 2017). Contudo, até o momento não há conhecimento e reflexão sobre a dimensão e as características da produção sobre a GE nos programas de pós-graduação brasileiros. A reflexão sobre esse cenário nos levou aos seguintes questionamentos: qual é o panorama atual da produção, onde as teses são desenvolvidas, quais temáticas são tratadas, qual seria a contribuição dessa produção para a solidificação e a qualidade, em termos acadêmicos, da GE no Brasil?

Entendemos que a análise destas produções pode auxiliar a compreender a maturidade da pesquisa na área, visto que as teses supostamente devem refletir a qualidade científica de um campo de estudo (ALMEIDA; GUIMARÃES, 2017; ATALAY, 2018), no caso a GE.

Diante do exposto, o objetivo da pesquisa foi analisar a produção de teses de doutorado em GE defendidas em programas de pós-graduação de IES brasileiras sob a ótica das suas temáticas e dos métodos de pesquisa utilizados.

\section{PROCEDIMENTOS METODOLÓGICOS}

A pesquisa se caracteriza como de caráter exploratório, descritivo e de abordagem mista, com característica de um projeto paralelo convergente (CRESWELL; CLARK, 2013; LI; PITTS; QUARTERMAN, 2008), por meio de uma revisão integrativa do tipo metodológica (WHITTEMORE, 2005).

A busca das teses em GE produzidas no Brasil até 2019 ocorreu nas seguintes bases de dados: Catálogo de Teses e Dissertações da Capes e a Biblioteca Digital Brasileira de Teses e Dissertações. Os termos de busca para a identificação dos documentos (teses) foram "gestão", "administração", "economia", "marketing" e "política" combinados com "esporte", "esportivo" e "esportiva".

Foram encontrados 894 trabalhos e a amostra foi constituída segundo critérios de exclusão aplicados em etapas: 1) análise das teses com registros repetidos nas bases de dados e de registros fora no escopo da GE; e 2) registros que não possuíam acesso ao trabalho completo.

A aplicação dos critérios da etapa 1 resultou em 106 teses e na etapa 2, em 12 registros não foi possível o acesso à versão completa. Como resultado, a amostra final foi constituída de 94 teses. 
Os dados de caracterização das teses, como título, ano, programa de pósgraduação, universidade e região geográfica foram organizados em planilha. Os resultados foram tabulados, enumerados e analisados posteriormente por meio da Estatística Descritiva (frequência).

Os arquivos das teses foram reunidos em uma pasta no Google Drive para possibilitar aos autores da pesquisa a análise dos dados relativos às temáticas $e$ aos métodos de pesquisa. Ambas as análises foram realizadas por meio da Análise de Conteúdo (BARDIN, 2006), utilizando-se a técnica de categorização (qualitativa - unidades de registro e contexto) e de codificação por unidade de enumeração (quantificação - frequência de ocorrência da unidade de registro).

Para construir a compreensão sobre as temáticas, utilizou-se a abordagem indutivo-construtiva, com categorias criadas a posteriori, dado que existe diversidade de entendimento com relação às áreas da $\mathrm{GE}$, além da área ser multidisciplinar em sua natureza.

No sentido de maximizar o grau de confiabilidade da categorização, o processo se deu com a análise inicial de dois dos autores, de forma independente, a partir da leitura do resumo, do sumário e da fundamentação teórica de cada tese. Posteriormente, o terceiro autor reavaliou os trabalhos, a partir dos mesmos procedimentos, para a construção do consenso (QUEIRÓS; GRAÇA, 2013).

Para a análise dos procedimentos metodológicos das teses, foi realizada a leitura completa, pelos autores da pesquisa, dos documentos, e a identificação das informações referentes a cinco categorias de análise. As categorias foram definidas a priori com base em autores teóricos de metodologia da pesquisa relacionados, ou não, à GE (EDWARDS; SKINNER, 2009; GIL, 2006; LI; PITTS; QUARTERMAN, 2008; MARCONI; LAKATOS, 2005; SKINNER; EDWARDS; CORBETT, 2015; VEAL; DARCY, 2014): presença de seção, item ou capítulo sobre os métodos utilizados; abordagem metodologia; método de pesquisa; método de coleta dos dados; e método de análise dos dados. Os dados obtidos foram quantificados, categorizados e analisados de forma qualitativa (BARDIN, 2006).

\section{RESULTADOS E DISCUSSÃO}

Com base nos procedimentos metodológicos, as 94 teses foram analisadas sob três perspectivas: caracterização da amostra, temáticas das teses e procedimentos metodológicos.

\subsection{CARACTERIZAÇÃO DAS TESES ANALISADAS}

Iniciando pelo ano em que as teses foram defendidas, a mais antiga data de 1997, havendo uma produção constante a partir de 2003, ainda que com a quantidade anual limitada. De 2013 em diante, exceção a 2015, é possível constatar aumento da produção, com pico de 14 trabalhos em 2014 (Gráfico 1). 
Gráfico 1 - Evolução anual da quantidade de teses finalizadas sobre Gestão do Esporte no Brasil (1997-2019)

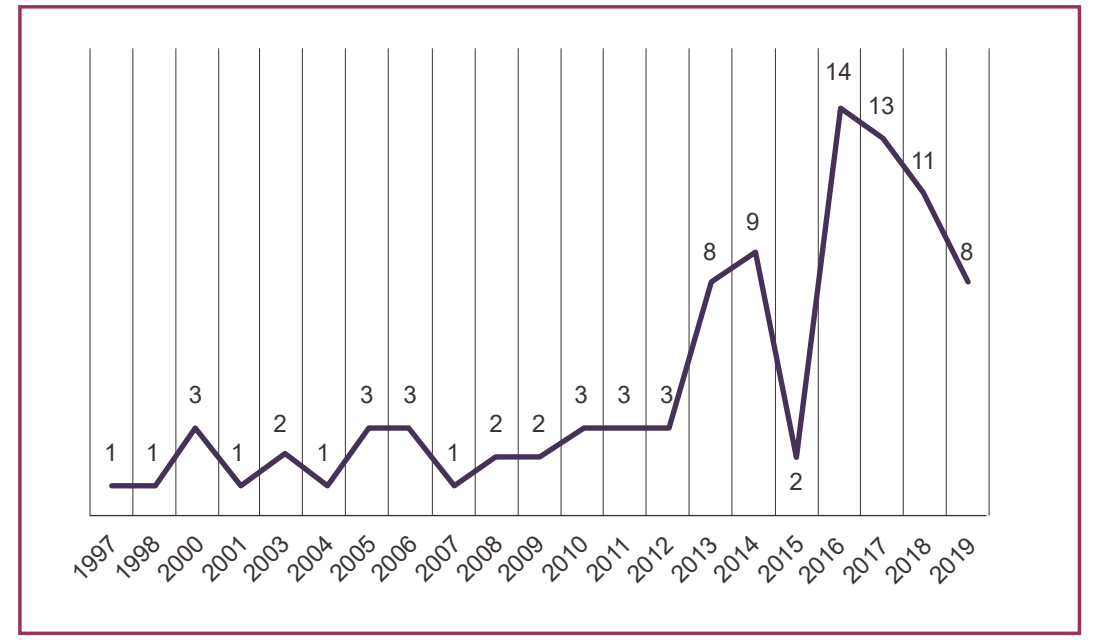

Fonte: dados da pesquisa

Os resultados, especialmente o crescimento a partir de 2008, são condizentes com a indicação de que a GE entrou em um período de crescimento a partir de 2009 devido a fatores como a escolha do Brasil para sediar megaeventos internacionais e o desenvolvimento acadêmico da área, como com a criação de grupos de estudos específicos (BASTOS, 2016; FRANCALACCI, 2011).

Sobre a região geográfica das IES em que as teses foram produzidas, foi verificado que $69 \%$ (65 trabalhos) foram realizadas no Sudeste, 14\% (13) no Sul, 10\% (9) no Nordeste e 7\% (7) no Centro-Oeste, números coerentes com a distribuição da produção científica do país (MANOEL; CARVALHO, 2011; ROJO; MEZZADRI; SILVA, 2019; SANTOS et al., 2012). Nenhuma tese sobre a GE foi localizada na Região Norte, revelando uma defasagem do desenvolvimento da área em instituições dessa região.

Dos 26 estados mais o Distrito Federal, dez tinham instituições com teses sobre GE (Tabela 1), com destaque para a concentração em São Paulo, com quantidade semelhante à soma dos demais. Em todos estados do Sul há teses, enquanto no Nordeste a produção acontece em três estados (Bahia, Pernambuco e Rio Grande do Norte) e no Centro-Oeste, no Distrito Federal.

Tabela 1 - Produção de teses por estado do país

\begin{tabular}{ccc}
\hline Estado & Quantidade & Porcentagem \\
\hline São Paulo & 45 & $48 \%$ \\
Rio de Janeiro & 13 & $14 \%$ \\
Distrito Federal & 7 & $7 \%$ \\
Minas Gerais & 7 & $7 \%$ \\
Rio Grande do Sul & 6 & $6 \%$ \\
Paraná & 6 & $6 \%$ \\
Bahia & 5 & $5 \%$ \\
Pernambuco & 3 & $3 \%$ \\
Rio Grande do Norte & 1 & $1 \%$ \\
Santa Catarina & 1 & $1 \%$ \\
\hline Total & 94 & $\mathbf{1 0 0 \%}$
\end{tabular}


A comparação dos dados com a distribuição dos programas de pós-graduação da área de Educação Física indicados por Corrêa, Corrêa e Rigo (2019), que revelam uma concentração no Sudeste (11 programas com doutorado) e no Sul (8), uma pequena quantidade no Centro-Oeste (2) e no Nordeste (1) e a ausência no Norte, poderia justificar a ausência de teses sobre a GE nessa região (CORRÊA; CORRÊA; RIGO, 2019).

Sobre as IES nas quais foram produzidas as teses, foi identificado um total de 28, sendo 19 públicas (68\%) e 9 privadas (32\%). Das 94 teses, 20 foram realizadas em entidades particulares e 74 em públicas (Quadro 1).

Quadro 1 - Quantidade de teses por instituições identificadas

\begin{tabular}{|c|c|c|c|c|c|}
\hline Instituição & $n^{0}$ & Instituição & $n^{0}$ & Instituição & $n^{\circ}$ \\
\hline $\begin{array}{c}\text { Universidade Estadual } \\
\text { de Campinas (SP) }\end{array}$ & 15 & $\begin{array}{l}\text { Pontifícia Universidade } \\
\underline{\text { Católica de São Paulo }}\end{array}$ & $\underline{4}$ & $\begin{array}{c}\text { Universidade de } \\
\text { Pernambuco }\end{array}$ & 1 \\
\hline $\begin{array}{l}\text { Universidade de } \\
\text { São Paulo }\end{array}$ & 12 & $\begin{array}{c}\text { Universidade do Estado } \\
\text { do Rio de Janeiro }\end{array}$ & 4 & $\begin{array}{l}\text { Universidade do Vale } \\
\underline{\text { do Rio dos Sinos (RS) }}\end{array}$ & 1 \\
\hline Universidade de Brasília & 6 & $\frac{\text { Fundação Getulio Vargas }}{\underline{\text { (SP e RJ) }}}$ & $\underline{3}$ & $\begin{array}{l}\text { Universidade Federal } \\
\text { de Juiz de Fora (MG) }\end{array}$ & 1 \\
\hline $\begin{array}{l}\text { Universidade Estadual } \\
\text { Paulista }\end{array}$ & 5 & $\frac{\text { Universidade São Judas }}{\underline{\text { Tadeu (SP) }}}$ & $\underline{3}$ & $\begin{array}{l}\text { Universidade Federal } \\
\text { de Lavras (MG) }\end{array}$ & 1 \\
\hline $\begin{array}{c}\text { Universidade Federal da } \\
\text { Bahia }\end{array}$ & 5 & $\begin{array}{c}\text { Universidade Federal de } \\
\text { Pernambuco }\end{array}$ & 2 & $\begin{array}{c}\text { Universidade Federal } \\
\text { de Santa Catarina }\end{array}$ & 1 \\
\hline $\begin{array}{c}\text { Universidade Federal de } \\
\text { Minas Gerais }\end{array}$ & 5 & $\begin{array}{l}\text { Universidade Federal do } \\
\text { Rio de Janeiro }\end{array}$ & 2 & $\begin{array}{l}\text { Universidade Federal } \\
\text { de São Carlos (SP) }\end{array}$ & 1 \\
\hline $\begin{array}{c}\text { Universidade Federal do } \\
\text { Paraná }\end{array}$ & 5 & $\frac{\text { Universidade Metodista }}{\underline{\text { de São Paulo }}}$ & $\underline{2}$ & $\begin{array}{c}\text { Universidade Federal } \\
\text { do Rio Grande do } \\
\text { Norte }\end{array}$ & 1 \\
\hline $\begin{array}{c}\text { Universidade Federal do } \\
\text { Rio Grande do Sul }\end{array}$ & 5 & $\begin{array}{c}\text { Instituto Tecnológico de } \\
\text { Aeronáutica (SP) }\end{array}$ & 1 & $\frac{\text { Universidade Nove de }}{\underline{\text { Julho }(\mathrm{SP})}}$ & 1 \\
\hline$\frac{\text { Universidade Gama }}{\underline{\text { Filho }(\mathrm{RJ})}}$ & $\underline{5}$ & $\frac{\text { Universidade Católica de }}{\underline{\text { Brasília }}}$ & 1 & $\begin{array}{c}\text { Universidade } \\
\text { Tecnológica Federal do } \\
\text { Paraná }\end{array}$ & 1 \\
\hline
\end{tabular}

Fonte: dados da pesquisa.

Observação: grifos nas instituições particulares.

Destacamos a produção nas três instituições públicas paulistas (Universidade Estadual de Campinas, Universidade de São Paulo e Universidade Estadual Paulista) que reúnem $34 \%$ dos trabalhos identificados. Algumas universidades federais respondem por grande parte das teses dos seus estados, como a Universidade de Brasília, a da Bahia, a de Minas Gerais, a do Paraná e a do Rio Grande do Sul. Com relação às instituições particulares, cinco são de São Paulo, duas do Rio de Janeiro, uma do Distrito Federal e uma do Rio Grande do Sul.

Ao comparamos os anos de defesa das teses com as instituições, pode ser constatado que as mais antigas, anteriores à produção constante a partir de 2003, estavam concentradas na Universidade Estadual de Campinas (3) e na Universidade de São Paulo (2), com uma maior diversificação de instituições a partir de 2016. Nas instituições privadas, a produção teve início em 2003 com uma concentração maior entre 2016 e 2018, evidenciando o crescimento da área indicado por Bastos (2016).

Sobre o formato adotado pelas teses, foram identificados dois modelos: 1) "tradicional", no qual é realizada uma pesquisa que visa defender um pressuposto 
ou hipótese, e 2) baseado em artigos, também denominado modelo escandinavo, no qual há um capítulo introdutório seguido por uma série de artigos - variando entre dois e cinco - e um capítulo conclusivo. A adoção do modelo escandinavo passou a ser incentivada visando sintetizar as teses e facilitar a leitura e a disseminação do conhecimento por meio dos artigos, tendência estimulada por universidades de diversos países (ABDOLMALAKI et al., 2019; NASSI-CALÒ, 2016; RUFINO, 2015).

Das 94 teses analisadas, 90\% adotaram o modelo "tradicional" e 10\% o escandinavo. Sobre as últimas, a primeira é de 2003, e houve crescimento da adoção do modelo após 2016 (7 teses), majoritariamente em programas de Educação Física (7), em diferentes instituições, principalmente no Sudeste (6), com destaque para a Universidade Estadual Paulista e Universidade Estadual do Rio de Janeiro. Das nove que utilizaram o modelo escandinavo, oito se valeram de artigos e uma de artigos e capítulo de livro.

Os dados permitem identificar que o uso do modelo escandinavo é uma tendência recente nas teses em GE indo ao encontro da literatura que indica a necessidade do aumento da publicação de artigos; difusão das pesquisas (NASSICALÓ, 2016; PALTRIDGE, 2002; RUFINO, 2015).

Por fim, com relação à área acadêmica dos programas de pós-graduação em que as teses foram produzidas, foram detectadas 14 áreas (Quadro 2). Há um predomínio da área de Educação Física, com $54 \%$ das teses, seguida pela Administração, com $17 \%$ dos trabalhos. Destaque ainda para a Educação e para as Ciências Sociais, com outras dez áreas representando 16\% das teses.

Quadro 2 - Área dos cursos de pós-graduação das teses analisadas

\begin{tabular}{|c|c|c|c|}
\hline Área & $\begin{array}{c}\text { Quantidade } \\
\text { de teses }\end{array}$ & Área & $\begin{array}{c}\text { Quantidade } \\
\text { de teses }\end{array}$ \\
\hline Educação Física & 51 & Política Social & 2 \\
\hline Administração & 16 & Antropologia Social & 1 \\
\hline Educação & 7 & Ciências da Computação & 1 \\
\hline Ciências Sociais & 5 & Engenharia Aeronáutica e Mecânica & 1 \\
\hline Engenharia de Produção & 4 & Sociologia & 1 \\
\hline Ciências da Saúde & 2 & Terapia Ocupacional & 1 \\
\hline Comunicação Social & 2 & - & - \\
\hline
\end{tabular}

Fonte: dados da pesquisa

Os resultados corroboram a multidisciplinariedade da GE, assim como a predominância do desenvolvimento dos seus estudos em IES relacionada à Educação Física/Esporte e à Administração, com destaque para os programas de pós-graduação em Educação Física, como também verificado no início do desenvolvimento da área nos Estados Unidos (CHALIP, 2006; SAWYER, 1993; WHITSON; MACINTOSH, 1990). O mesmo foi constatado no Brasil por Gaya; Gaya; Reppold Filho; Cardoso e Bento (2018) em estudo sobre teses brasileiras sobre o Esporte (GAYA et al., 2018).

A concentração do desenvolvimento das teses em GE nos programas de pós-graduação em Educação Física e Esporte de certa forma também pode 
estar relacionada a grupos de pesquisa da área no Brasil, que também possuem predominância de vínculo nas mesmas áreas (BASTOS, 2019).

\subsection{TEMÁTICA DAS TESES}

A Análise de Conteúdo das temáticas das teses resultou em 16 categorias (Quadro 3), com preponderância de Políticas Públicas (39\%), Marketing (12\%) e Eventos (10\%). Seguem-se as categorias Governança (6\%); Economia (5\%); esporte internacional (4\%) - relativo a temas esportivos globais como olimpismo e entidades esportivas internacionais - e Modalidades Brasil, sobre especificidades da GE de modalidades (4\%). As outras nove categorias se referem a 18 teses $(19 \%)$.

Quadro 3 - Categorias temáticas das teses analisadas

\begin{tabular}{|c|c|c|c|c|c|}
\hline Categoria & Quantidade & Categoria & Quantidade & Categoria & Quantidade \\
\hline $\begin{array}{l}\text { Políticas } \\
\text { Públicas }\end{array}$ & 37 & $\begin{array}{c}\text { Modalidades } \\
\text { Brasil }\end{array}$ & 4 & Ética & 2 \\
\hline Marketing & 11 & $\begin{array}{l}\text { Gestão de } \\
\text { carreiras }\end{array}$ & 3 & $\begin{array}{l}\text { Produção do } \\
\text { conhecimento }\end{array}$ & 2 \\
\hline Eventos & 9 & $\begin{array}{l}\text { Recursos } \\
\text { Humanos/ } \\
\text { Gestão de } \\
\text { Pessoas }\end{array}$ & 3 & Empreendedorismo & 1 \\
\hline Governança & 6 & Comunicação & 2 & Instalações & 1 \\
\hline Economia & 5 & $\begin{array}{c}\text { Direito/ } \\
\text { Legislação }\end{array}$ & 2 & - & - \\
\hline $\begin{array}{c}\text { Esporte } \\
\text { internacional }\end{array}$ & 4 & $\begin{array}{l}\text { Esporte } \\
\text { adaptado }\end{array}$ & 2 & - & - \\
\hline
\end{tabular}

Fonte: dados da pesquisa

Quando comparado com a produção internacional, há semelhanças no que diz respeito às temáticas das teses quanto a Marketing e Governança (CIOMAGA, 2013; LARA, 2014; PITTS; DANYLCHUCK; QUARTERMAN, 2014; PITTS; PEDERSEN, 2005; SHILBURY, 2011). A temática Eventos, que possui maior produção de teses a partir de 2013, provavelmente em resposta à candidatura e realização de megaeventos esportivos no Brasil, também possui destaque na literatura internacional (CIOMAGA, 2013; LARA, 2014).

A temática Políticas Públicas é a de maior destaque entre as teses analisadas. Esta particularidade pode ser justificada tendo em vista a dependência do investimento governamental no desenvolvimento do esporte, uma peculiaridade da Indústria do Esporte nacional (MAZZEI; ROCCO JÚNIOR, 2017).

Também corroboram os dados internacionais o baixo desenvolvimento de teses nas categorias Direito/Legislação, Ética, Comunicação, Instalações Esportivas (CIOMAGA, 2013; PITTS; DANYLCHUCK; QUARTERMAN, 2014; PITTS; PEDERSEN, 2005), temáticas também identificadas como lacuna nos currículos de cursos de graduação em Educação Física/Esporte (BASTOS, 2016; QUINAUD; FARIAS; NASCIMENTO, 2018). 
É possível identificar que nas 29 teses produzidas até 2012, período no qual a quantidade anual de teses era menor, a principal temática era Políticas Públicas (11 teses), seguida por Marketing (4), Economia (3) e modalidades esportivas do Brasil (3). A partir de 2013, quando houve um crescimento da produção, a maioria das teses trata de Políticas Públicas (26), mas com crescimento de Marketing (7), Governança (5) e esporte internacional (3) e identificação de novas temáticas, especialmente Eventos (9) e Gestão de Carreiras (3).

A interpretação conjunta das temáticas e da produção por regiões do Brasil revela convergências, como o fato de que na região Centro-Oeste a produção é focada em Políticas Públicas, com quatro teses de um total de sete. Esse mesmo cenário é identificado no Nordeste, onde oito de nove teses abortam tal temática. Por outro lado, no Sul é verificada uma diversidade, com sete diferentes temáticas, apesar do predomínio das Políticas Públicas (4 de 13). No Sudeste, região com maior quantidade de teses, a maioria é Políticas Públicas (21 de 37), Marketing (8 de 11) e Governança (5 de 6). Ressalta-se que as teses sobre Ética são todas da Universidade Federal do Rio Grande do Sul e que todas as teses sobre esporte internacional, Gestão de Carreiras, Recursos Humanos/Gestão de Pessoas e Comunicação foram desenvolvidas no Sudeste.

Considerando as teses produzidas em instituições privadas (20), foi constatado que as principais temáticas são Políticas Públicas (4 teses), Eventos (4 de um total de 9), Governança (3 de 6), Recursos Humanos/Gestão de Pessoas (2 de 3) e Comunicação (todas).

A interpretação da relação entre as áreas dos programas de pós-graduação com as temáticas evidencia que o estudo de Políticas Públicas é desenvolvido em programas de Educação Física e os de Marketing se concentram na Administração (Quadro 4).

Quadro 4 - Temáticas pesquisados nas teses segundo as principais áreas dos programas de pósgraduação

\begin{tabular}{|c|c|c|}
\hline Área dos programas & \multicolumn{2}{|c|}{ Temáticas } \\
\hline Educação Física & Políticas Públicas (23) & Eventos (5) \\
\hline Administração & Marketing (5) & Governança (3) \\
\hline Educação & \multicolumn{2}{|c|}{ Todas são de Políticas Públicas (7) } \\
\hline Ciências Sociais & Governança (2) & Eventos Esportivos (2) \\
\hline
\end{tabular}

Fonte: dados da pesquisa

A partir dos resultados e das interpretações conjuntas realizadas podemos indicar características sobre o desenvolvimento das teses que tratam das principais temáticas identificadas. As que abordam as Políticas Públicas apresentam uma produção constante, com o volume proporcional do Nordeste e do Centro-Oeste superior ao do Sudeste e Sul e foram desenvolvidas em programas de pós-graduação de Educação Física de instituições públicas: 33 teses das 37. As relativas a Marketing foram realizadas, majoritariamente, após 2013, principalmente no Sudeste e em programas de Administração de instituições públicas (9 de 11 teses).

Sobre as teses que tratam de Eventos, elas se concentram após 2013, no Sudeste e em programas de Educação Física, havendo um equilibro entre as 
IES privadas e públicas. Por fim, as teses que tratam de Governança apresentam características semelhantes das sobre Marketing no que se refere ao período, região e área do programa, se diferenciando por estarem distribuídas de forma equilibrada entre instituições públicas e privadas.

\subsection{MÉTODOS UTILIZADOS}

Os métodos foram analisados e apresentados segundo as cinco categorias elencadas nas decisões metodológicas.

\subsubsection{Seção, item ou capítulo específico sobre os procedimentos metodológicos}

A verificação da existência ou não de seção, item ou capítulo específico detalhando os procedimentos metodológicos nas 94 teses resultou em 69 (73\%) apresentando, enquanto que 25 (27\%) não. Todas que não possuíam eram do formato "tradicional", sendo que em sete das nove teses que adotaram o modelo escandinavo tal item/capítulo estava inserido dentro dos artigos, e em duas havia também um item geral sobre a metodologia.

A ausência dessa seção, item ou capítulo, tidos como fundamental no modelo "tradicional" (ABDOLMALAKI et al., 2019; PALTRIDGE, 2002), dificulta a identificação e a compreensão dos procedimentos metodológicos realizados na pesquisa, bem como a sua replicação (SKINNER; EDWARDS; CORBETT, 2015).

\subsubsection{Abordagem metodológica}

Com relação à abordagem metodológica indicada pelos autores das teses, foi observado que $48(51 \%)$ utilizaram a qualitativa, três (3\%) a quantitativa e 16 (17\%) a mista, sendo que em 27 teses (29\%) tal informação não foi apresentada.

Destaque para o limitado número de trabalhos quantitativos, sendo eles sobre Políticas Públicas (2) e Marketing (1), e para o fato de quase um terço das teses não citaram claramente tal informação, a maior parte da área de Políticas Públicas (13) e de programas de Educação Física (14), informação importante para caracterizar a pesquisa realizada (EDWARDS; SKINNER, 2009).

O elevado número de pesquisas de abordagem qualitativa pode ser explicado pela GE ainda ser uma disciplina em desenvolvimento no Brasil (Bastos, 2016), de forma que seus estudos tendem a ser exploratórios da realidade, abordagem mais adequada para esses fins (EDWARDS; SKINNER, 2009).

Ao compararmos com a literatura internacional, notamos percurso diferente, pois há tendência quantitativa histórica nas publicações internacionais (PITTS; PEDERSEN, 2005; SKINNER; ENGELBERG, 2018). Essa tendência também foi observada por Castro, Silva e Lüdorf (2019) ao analisarem a produção de teses nos programas de pós-graduação brasileiros em Educação Física. Há uma clara distinção entre a principal opção adotada pela área da GE (qualitativa) e a utilizada por outras áreas da Educação Física, como Fisiologia e Biomecânica, que costumeiramente adotam a abordagem quantitativa. 


\subsubsection{Método de pesquisa}

O método utilizado para a realização das pesquisas nas teses foi citado em 76 (81\%), em 18 (19\%) não. Identificamos a utilização de 21 métodos, com a utilização de mais de um em parte dos trabalhos $(31 \%)$. O número de citações de métodos foi 116 e no Quadro 5 são apresentados aqueles com pelo menos dez citações.

Quadro 5 - Métodos de pesquisa mais utilizados nas teses analisadas

\begin{tabular}{|c|c|c|c|}
\hline Método & $\begin{array}{c}\text { Quantidade de } \\
\text { citações }\end{array}$ & Método & $\begin{array}{c}\text { Quantidade de } \\
\text { citações }\end{array}$ \\
\hline Pesquisa Documental & 23 & Survey & 11 \\
\hline Entrevista & 21 & Pesquisa Bibliográfica & 10 \\
\hline Estudo de Caso & 19 & Outros (16 métodos) & 32 \\
\hline
\end{tabular}

Fonte: dados da pesquisa.

Quanto à utilização de base teórica para embasar o método de pesquisa utilizado, $67 \%$ das teses apresentam referências de autores, enquanto que 33\% não citaram, o que limita a análise dos métodos e procedimentos realizados (SKINNER; EDWARDS; CORBETT, 2015).

\subsubsection{Método de coleta de dados}

Quanto ao método utilizado para a coleta das informações que subsidiaram as pesquisas, em 85 trabalhos os autores indicaram um ou mais métodos, enquanto que em nove tal informação não foi apresentada.

Ao todo foram identificados seis métodos, com grande parte das pesquisas $(72 \%)$ se valendo de mais de um, com um total de menções de 182 (Quadro 6). Destaque para a Pesquisa Documental e a Entrevista e a menor utilização de Grupo Focal.

Quadro 6 - Métodos de coletada de dados citados nas teses analisadas.

\begin{tabular}{|c|c|c|c|}
\hline Método & Quantidade de citações & Método & Quantidade de citações \\
\hline Pesquisa Documental & 57 & Questionário & 21 \\
\hline Entrevista & 55 & Observação & 18 \\
\hline Pesquisa Bibliográfica & 28 & Grupo focal & 3 \\
\hline
\end{tabular}

Fonte: dados da pesquisa.

Os métodos Pesquisa Documental e Pesquisa Bibliográfica, através dos quais se obtêm dados secundários, foram identificados em 16 teses, que se valeram exclusivamente deles. Métodos de obtenção de dados primários, ou de campo, estão presentes em $83 \%$ dos trabalhos.

\subsubsection{Método de análise dos dados}

Com relação aos métodos utilizados para analisar os dados, em 71 teses (76\%) os autores os indicaram claramente, enquanto que em 23 (24\%), não. Ao 
todo foram identificados 33 métodos, com algumas teses aplicando mais de um, e verificadas 96 citações de métodos (Quadro 7).

Quadro 7 - Métodos de análise dos dados citados nas teses analisadas.

\begin{tabular}{|c|c|c|c|}
\hline Método & $\begin{array}{c}\text { Quantidade de } \\
\text { citações }\end{array}$ & Método & $\begin{array}{c}\text { Quantidade de } \\
\text { citações }\end{array}$ \\
\hline Análise de Conteúdo & 29 & Método Comparativo & 6 \\
\hline Métodos estatísticos & 23 & Análise do Discurso & 4 \\
\hline $\begin{array}{c}\text { Categorização/classificação } \\
\text { dos dados }\end{array}$ & 7 & Outros (16 métodos) & 17 \\
\hline
\end{tabular}

Fonte: dados da pesquisa.

Sobre os métodos estatísticos, nenhuma tese utilizou técnicas probabilísticas, 15 se valeram de técnicas descritivas e 12 trabalhos de 18 técnicas inferenciais (alguns utilizaram em mais de um), com destaque para Análise Fatorial, Modelagem de Equações Estruturadas e Regressão, cada uma citada em três trabalhos distintos.

Entre os métodos citados, os mais utilizados foram os qualitativos (54\%), seguidos pelos quantitativos (34\%) e pelos mistos (11\%), com uma maior variedade de métodos qualitativos (15) e predomínio da utilização da Análise de Conteúdo.

Ao comparamos a quantidade de métodos de análise quantitativos identificados com o fato de que apenas três teses indicaram possuírem abordagem metodológica quantitativa podemos observar uma discrepância entre tais resultados. Essa diferença pode ser justificada devido ao elevado número de teses que não indicaram a abordagem (29\%), por descuido dos pesquisadores, pela utilização de abordagens mistas ou até mesmo pela classificação errônea.

Quanto à indicação de referências teóricas para embasar os métodos de análise de dados, considerando apenas as 71 teses que indicaram métodos de análise, $83 \%$ citaram referências e $17 \%$ não.

Por fim, destaque para o fato de que uma tese não apresenta nenhuma das informações analisadas sobre os procedimentos metodólogos utilizados; uma cita só a abordagem; quatro somente o método; duas apenas o método de coleta dos dados; e duas indicam unicamente o método de análise de dados.

Ainda que alguma variedade de métodos de pesquisa, instrumentos de coleta de dados e métodos de análise tenham sido identificada, é possível considerar que há a necessidade marcante de maior rigor científico na pesquisa acadêmica em GE no Brasil. Resultados semelhantes foram encontrados em trabalhos da GE de outro país onde a área também é recente (ATALAY, 2018).

Nesse sentido, Amis e Silk (2005) afirmam que a área exige um arsenal metodológico expansivo e reflexivo, de forma a compreender o campo amplo que é a GE, contribuindo com o desenvolvimento de novas possibilidades de pesquisa e uma democracia de abordagens. Sem a variedade de abordagens, os pesquisadores podem assumir que os métodos já amplamente utilizados não possuem falhas ou não podem ser aprimorados (HOEBER; SHAW, 2017), o que pode prejudicar a expansão e desenvolvimento científico da área. 


\section{CONCLUSÃO}

O levantamento e a análise da produção de teses de doutorado em GE defendidas em programas de pós-graduação brasileiros em relação às temáticas abordadas e aos métodos de pesquisa empregados forneceram um panorama da produção acadêmica sobre a área no país.

A caracterização das teses revela que o desenvolvimento é relativamente recente, com a distribuição geográfica irregular pelo Brasil e majoritariamente produzidas em programas de universidades públicas, nas áreas de Educação Física e Administração. As teses sobre GE se desenvolvem em programas de pós-graduação de diferentes áreas, reafirmando o caráter multidisciplinar da Gestão do Esporte. Quanto às temáticas, identificou-se uma diversidade, apesar de limitada frente às encontradas nas produções internacionais da GE.

Foram verificados pontos a serem aprimorados em relação às estruturas metodológicas das teses brasileiras de doutorado em GE. As principais limitações se referem à falta de informações relevantes, como abordagem, método de pesquisa e de análise, além da ausência de bases teóricas que os fundamentem. Estes problemas se configuram como um fator crucial que pode levar a questionamentos quanto à confiabilidade e à fidedignidade das pesquisas acadêmicas da área. Além disso, essa omissão dificulta o entendimento dos leitores e pesquisadores quanto aos procedimentos realizados nas pesquisas, comprometendo a sua reprodutibilidade. Tais limitações metodológicas podem ser justificadas pela ausência de obras sobre a metodologia da pesquisa aplicada à GE na literatura brasileira.

Como limitações da presente pesquisa, indicamos dificuldades na busca das teses devido a problemas nas bases de dados, como ausência de mecanismo de refinamento das buscas e a indisponibilidade do acesso à versão integral de alguns registros. Outra limitação foi a dificuldade de identificar e analisar os itens metodológicos e padronizá-los a partir dos parâmetros definidos a priori, já que há diferentes características nas áreas de conhecimento dos programas em que as teses foram desenvolvidas.

Como implicações teóricas indicamos que a pesquisa expôs o estado da arte sobre a produção de teses em GE no Brasil, sintetizando o conhecimento de uma área emergente e complexa e possibilitando nortear novas pesquisas, além de ser mais um passo no processo de consolidação da área. Já como implicações práticas, é evidenciada a diversidade temática e metodológica da GE, subsidiando os pesquisadores da área na realização de novos estudos.

Para o desenvolvimento científico daárea recomenda-se aprodução de literatura sobre metodologia da pesquisa aplicada à GE que possa auxiliar pesquisadores a embasarem seus trabalhos científicos. Outra sugestão é o desenvolvimento de pesquisas que supram as lacunas identificadas quanto às áreas temáticas, além de estudos quantitativos e a utilização de outros métodos de pesquisa, coleta e análise dos dados.

Como sugestões de estudos futuros indicamos a continuidade das análises das teses no sentido de avaliar a evolução acadêmico-científica, bem como a análise 
da produção de dissertações de mestrado e de artigos científicos sobre temáticas da Gestão do Esporte no país.

\section{REFERÊNCIAS}

ABDOLMALAKI, Shahrbanou Ghorban; TAN, Helen; ABDULLAH, Ain Nadzimah; SHARMINI, Sharon; IMM, Lee Geok. Introduction chapter of traditional and article-based theses: A comparison of rhetorical structures and linguistic realisations. GEMA Online Journal of Language Studies, v. 19, n. 1, p. 116-135, 2019.

ALMEIDA, Elenara Chaves Edler; GUIMARÃES, Jorge Almeida (orgs.). A pós-graduação e a evolução da produção científica brasileira. São Paulo: SENAC, 2017.

AMIS, John; SILK, Michael. Ruptur: promoting critical and innovative approaches to the study of sport management. Journal of Sport Management, v. 19, p. 355-366, 2005.

ABRAGESP - ASSOCIAÇÃO BRASILEIRA DE GESTÃO DO ESPORTE. Quem somos. 2009. Disponível em: http://www.abragesp.org.br/quem-somos/. Acesso em: 23 maio 2020.

ATALAY, Ahmet. Research models used in doctoral theses on sport management in Turkey: a content analysis. Universal Journal of Educational Research, v. 6, n. 3, p. 541-555, 2018.

BARDIN, Laurence. Análise de conteúdo. Lisboa: Edições 70 Brasil, 2006.

BASTOS, Flávia da Cunha. Gestão do Esporte no Brasil: reflexões sobre avanços, limites e desafios. 2016. Tese (Livre-Docência) - Escola de Educação Física e Esporte, Universidade de São Paulo, 2016.

BASTOS, Flávia da Cunha. Sport management scientific development in Brazil. In: ZHANG, James; PITTS, Brenda (eds.). Globalized Sport Management in Diverse Cultural Contexts. Abingdon: Routledge, 2019. p. 136-153.

BASTOS, Flávia da Cunha; CASTRO, Claiton; AMARAL, Cacilda Mendes dos Santos. Marketing em academias no brasil: o abandono da academia. In: CONGRESSO BRASILEIRO SOBRE GESTÃO DO ESPORTE. 6., 2015. Anais [...]. Rio de Janeiro: ABRAGESP, 2015. Disponível em: http://www.abragesp.org.br/docs/Anais_CIGESP_2015. pdf. Acesso em: 22 maio 2020.

CASTRO, Pedro Henrique Zubcich Caiado; SILVA, Alan Camargo; LÜDORF, Sílvia Maria Agatti. Dissertações e Teses em Educação Física: uma investigação sobre abordagens metodológicas. Movimento, v. 25, e25013, 2019. DOI: https://doi.org/10.22456/1982$\underline{8918.82495}$

CHALIP, Laurence. Toward a distinctive sport management discipline. Journal of Sport Management, v. 20, n. 1, p. 1-21, 2006.

CHELLADURAI, Packianathan. Managing organizations for sport and physical activity: a systems perspective. $4^{\text {th }}$. ed. Scottsdale, AZ: Holcomb Hathaway , 2013.

CIOMAGA, Bogdan. Sport management: A bibliometric study on central themes and trends. European Sport Management Quarterly, v. 13, n. 5, p. 557-578, 2013. 
COSMA - COMMISSION ON SPORT MANAGEMENT ACCREDITATION. Accreditation principles manual \& guidelines for self-study preparation. Fort Collins Commission on Sport Management Accreditation, 2016. Disponível em: https://www.cosmaweb.org/ accreditation-manuals.html. Acesso em: 20 maio 2020.

CORRÊA, Marluce Rauel Decian; CORRÊA, Leandro Quadro; RIGO, Luiz Carlos. A pós-graduação na educação física brasileira: condições e possibilidades das subáreas sociocultural e pedagógica. Revista Brasileira de Ciências do Esporte, v. 41, n. 4, p. 359-366, 2019. DOI: https://doi.org/10.1016/j.rbce.2018.03.009.

CRESWELL, John W; CLARK, Vick L. Plano. Escolha de um projeto de métodos mistos. In: CRESWELL, John W; CLARK, Vick L. Plano (eds.). Pesquisa de Métodos Mistos. 2. ed. Porto Alegre: Penso, 2013. p. 60-103.

DOWLING, Mathew. Exploring sport management as an academic profession: a critical review of occupational theory. Journal of Global Sport Management, v. 3, n. 4, p. 321-338, 2018.

EDWARDS, Allan; SKINNER, James. Qualitative research in Sport Management. Hungary: Elsevier, 2009.

FRANCALACCI, Vanessa Lins. Desenvolvimento e estado da arte da Gestão do Esporte no Brasil, 1909-2009. 2011. Tese (Doutorado em Educação Física) - Universidade Gama Filho, Rio de Janeiro, 2011.

GAYA, Adroaldo Cezar; GAYA, Anelise Reis; REPPOLD FILHO, Alberto Reinaldo; CARDOSO, Vinícius Denardin; BENTO, Jorge Olímpio. Estudo descritivo sobre a ocorrência de teses sobre o esporte nos programas de pós-graduação no Brasil. Revista Brasileira de Ciências do Esporte, v. 40, n. 4, p. 346-352, 2018.

GIL, Antonio Carlos. Métodos e técnicas de pesquisa social. 5. ed. São Paulo: Atlas, 2006.

HOEBER, Larena; SHAW, Sally. Contemporary qualitative research methods in sport management. Sport Management Review, v. 20, n. 1, p. 4-7, 2017.

LARA, Felipe Ferreira. Evolução da literatura sobre gestão esportiva: Estudo bibliométrico em um período de vinte anos (1993 - 2012). Gestão Contemporânea, v. 16, p. 166-192, 2014.

LI, Ming; PITTS, Brenda; QUARTERMAN, Jerome. Research methods in sport management. Morgantown, WV: Fitness Information Technology, 2008.

MANOEL, Edison Jesus; CARVALHO, Yara Maria. Pós-graduação na Educação Física brasileira: a atração (fatal) para a biodinâmica. Educação e Pesquisa, v. 37, n. 2, p. 389406, 2011.

MARCONI, Marina; LAKATOS, Eva Maria. Fundamentos de Metodologia Científica. 6. ed. São Paulo: Editora Atlas, 2005.

MAZZEI, Leandro Carlos; AMAYA, Katherine; BASTOS, Flávia da Cunha. Programas acadêmicos de graduação em Gestão do Esporte no Brasil. Revista Mackenzie de Educação Física e Esporte, v. 12, n. 1, p. 219-234, 2013.

MAZZEI, Leandro Carlos; ROCCO JÚNIOR, Ary José. Um ensaio sobre a gestão do esporte : um momento para a sua afirmação no Brasil. Revista de Gestão e Negócios do Esporte (RGNE), v. 2, n. 1, p. 96-109, 2017. 
MIRAGAIA, Dina; SOARES, Jorge. Higher education in sport management: A systematic review of research topics and trends. Journal of Hospitality, Leisure, Sport \& Tourism Education, v. 21, pt. A, p. 101-116, Nov. 2017.

NASSI-CALÒ, Lilian. Teses e dissertações: prós e contras dos formatos tradicional e alternativo. SciELO em perspectiva, 2016. Disponível em: https://blog.scielo.org/ blog/2016/08/24/teses-e-dissertacoes-pros-e-contras-dos-formatos-tradicional-e-alternativo/. Acesso em: 23 abr. 2020.

PALTRIDGE, Brian. Thesis and dissertation writing: An examination of published advice and actual practice. English for Specific Purposes, v. 21, n. 2, p. 125-143, 2002.

PARKHOUSE, Bonnie L. The management of Sport: its foundation and application. 4 ed. New York: McGrow-Hill, 2005.

PIRES, Gustavo Manoel Vaz da Silva; SARMENTO, José Pedro. Conceito de gestão do desporto. novos desafios, diferentes soluções. Revista Portuguesa de Ciências do Desporto, v. 1, n. 1, p. 88-103, 2001.

PITTS, Brenda. Sport management at the millennium: a defining moment. Journal of Sport Management, v. 15, p. 1-9, 2001.

PITTS, Brenda; DANYLCHUCK, Karen; QUARTERMAN, Jerome. Analysis of sport management literature : european sport management quarterly. Sport Management International Journal, v. 10, n. 2, p. 45-72, 2014.

PITTS, Brenda; PEDERSEN, Paul. Examining the body of knowledge in sport management: A content analysis of the Journal of Sport Management. Sport Management and Related Topics Journal, v. 2, n. 1, p. 33-52, 2005.

QUEIRÓS, Paula; GRAÇA, Amândio. A Análise de Conteúdo (enquanto técnica de tratamento da informação) no âmbito da investigação qualitativa. In: MESQUITA, Isabel.; GRAÇA, Amândio (eds.). Investigação Qualitativa em Desporto. Porto: Universidade do Porto. Centro de Investigação Formação Inovação e Intervenção em Desporto, 2013. v. 1, p. 115-146.

QUINAUD, Ricardo Teixeira; FARIAS, Gelcemar Oliveira; NASCIMENTO, Juarez Vieira. Formação profissional do gestor esportivo para o mercado de trabalho: a (in)formação dos cursos de bacharelado em Educação Física do Brasil. Movimento, v. 24, n. 4, p. 1111-1124, 2018.

ROCHA, Cláudio Miranda; BASTOS, Flávia da Cunha. Gestão do Esporte: definindo a área. Revista Brasileira de Educação Física e Esporte, v. 25, n. esp, p. 91-103, 2011.

ROJO, Jeferson Roberto; MEZZADRI, Fernando Marinho; SILVA, Marcelo Moraes. A produção do conhecimento sobre políticas públicas para o esporte e lazer no Brasil: uma análise dos pesquisadores e instituições. PODIUM Sport, Leisure and Tourism Review, v. 8, n. 1, p. 128-139, 2019.

RUFINO, Luiz Gustavo Bonatto. Entre o modelo tradicional e o escandinavo de produção de tese. Pós-Graduando, 2015. Disponível em: https://tinyurl.com/yd7paxlk. Acesso em: 29 abr. 2020.

SAJJADI, S. N.; BEHNAM, M.; HASHEMI, S. Z.; BAKHSHANDEH, H.; AHMADI, H. R.. the evaluation of scientific-research articles on sports management in Iranian professional journals. Middle-East Journal of Scientific Research, v. 13, n. 10, p. 1413-1418, 2013. Disponível em: https://www.idosi.org/mejsr/mejsr13(10)13/19.pdf. Acesso em: 18 nov. 2019. 
SANTOS, Amanda; BASTOS, Letícia Lemos Ayres da Gama; ALEIXO, Andrezza Aparecida; PAULO, Thaís Reis Silva de; MENDES, Edmar Lacerda. Distribuição, evolução e produção científica dos grupos de pesquisa em atividade física e saúde do Brasil. Revista Brasileira de Atividade Física \& Saúde, v. 17, n. 4, p. 258-262, 2012.

SAWYER, Thomas H. Editorial. Sport management: where should it be housed? Journal of Physical Education, Recreation \& Dance, v. 64, n. 9, p. 4-5, 1993.

SCELLES, Nicolas. International dynamics of sport management: Towards an Agenda 21. [ID] hal02498272. Disponível em: https://hal.archives-ouvertes.fr/hal-02498272/ Acesso em: 25 abr. 2020.

SHILBURY, David. A bibliometric analysis of four sport management journals. Sport Management Review, v. 14, n. 4, p. 434-452, 2011.

SKINNER, James; EDWARDS, Allan; CORBETT, Ben. Research Methods for Sport Management. New York: Routledge, 2015.

SKINNER, James; ENGELBERG, Terry. New problems, new challenges: embracing innovative approaches to sport research. Sport in Society, v. 21, n. 2, p. 179-184, 2018.

SLACK, Trevor. The social and commercial impact of sport, the role of sport management. European Sport Management Quarterly, v. 14, n. 5, p. 454-463, 2014.

SMITH, Aaron C. T.; STEWART, Bob. The special features of sport: a critical revisit. Sport Management Review, v. 13, n. 1, p. 1-13, 2010.

VEAL, Anthony James; DARCY, Simon. Research methods in sport studies and sport management: A Practical Guide. New York: Routledge, 2014.

WHITSON, David. J.; MACINTOSH, Donald. the scientization of physical education: discourses of performance. Quest, v. 42, n. 1, p. 40-51, 1990.

WHITTEMORE, Robin. Combining evidence in nursing research: methods and implications. Nursing Research, v. 54, n. 1, p. 56-62, 2005.

YIAMOUYIANNIS, Athena; BOWER, Glenna G.; WILLEAMS, Joanne; GENTILE, Dina; ALDERMAN, Heather. Sport Management Education: accreditation, accountability, and direct learning outcome assessments. Sport Management Education Journal, v. 7, n. 1, p. 51-59, 2013. 
Abstract: This study analyzes themes and research methods adopted in doctoral theses on Sport Management at graduate programs of Brazilian HEls. The exploratory, descriptive and mixed-approach research was carried out through a methodological integrative review, considering theses defended until 2019. 94 theses were found, starting in 1997, with uneven geographic distribution throughout the country, mostly produced in public universities' Physical Education and Administration programs. The theses present a range of themes where public policies prevail, while there are gaps regarding other research topics. Critical aspects were revealed about the methodological structure, with a lack of information regarding the approach and the research and analysis methods. The panorama of academic production in the area reveals gaps consistent with those of its scientific development in Brazil.

Keywords: Management. Sports. Electronic Thesis. Scientific Publication Indicators.

Resumen: El presente estudio tuvo como objetivo analizar temas y métodos de investigación en la producción de tesis doctorales en Gestión Deportiva en programas de posgrado de IES brasileñas. La investigación, de enfoque exploratorio, descriptivo y mixto, se realizó a través de revisión integradora de tipo metodológica, considerando tesis defendidas hasta 2019. Se identificaron 94 tesis, a partir de 1997, con distribución geográfica desigual en el país, producidas principalmente en universidades públicas, en programas de Educación Física y Administración. Las tesis presentan diversidad de temas, con predominio de políticas públicas y déficit de otros temas de investigación. Se revelaron aspectos críticos con respecto a la estructura metodológica, con falta de información acerca del enfoque y los métodos de investigación y análisis. Se concluye que el panorama de la producción académica en el área revela retrasos coherentes con los del desarrollo científico del área en el país.

Palabras clave: Gestión. Deportes. Tesis Electrónicas. Indicadores de Producción Científica. 


\section{LICENÇA DE USO}

Este é um artigo publicado em acesso aberto (Open Access) sob a licença Creative Commons Atribuição 4.0 Internacional (CC BY 4.0), que permite uso, distribuição e reprodução em qualquer meio, desde que o trabalho original seja corretamente citado. Mais informações em: http://creativecommons.org/licenses/by/4.0

\section{CONFLITO DE INTERESSES}

Os autores declararam que não há conflito de interesses neste trabalho.

\section{CONTRIBUIÇÕES AUTORAIS}

Ivan Furegato Moraes: Responsável pela coleta, análise e discussão dos dados.

Cacilda Mendes dos Santos Amaral: Responsável pela fundamentação teórica e pela coleta e discussão dos dados.

Flávia da Cunha Bastos: Responsável pela fundamentação teórica, procedimentos metodológicos e discussão dos dados.

\section{FINANCIAMENTO}

O presente trabalho foi realizado com apoio da Coordenação de Aperfeiçoamento de Pessoal de Nível Superior -Brasil (CAPES) - Código de Financiamento 001 This study was financed in part by the Coordenação de Aperfeiçoamento de Pessoal de Nível Superior - Brasil (CAPES) - Finance Code 001.

\section{COMO REFERENCIAR}

MORAES, Ivan Furegato; AMARAL, Cacilda Mendes dos Santos; BASTOS , Flávia da Cunha. Teses de doutorado em gestão do esporte no brasil: uma revisão integrativa metodológica. Movimento (Porto Alegre), v.27, e27012, jan./dez. 2021. Disponível em: https://www.seer.ufrgs.br/Movimento/article/view/103915. Acesso em: [dia] [mês abreviado]. [ano]. DOI: https://doi.org/10.22456/1982$\underline{8918.103915}$

\section{RESPONSABILIBADE EDITORIAL}

Alex Branco Fraga*, Elisandro Schultz Wittizorecki, Ivone Job*, Mauro Myskiw*, Raquel da Silveira*

*Universidade Federal do Rio Grande do Sul, Escola de Educação Física, Fisioterapia e Dança, Porto Alegre, RS, Brasil 